\title{
Characteristics of Endurance Competitions and Risk Factors for Elimination in New Zealand during Six Seasons of Competition (2010/11-2015/16)
}

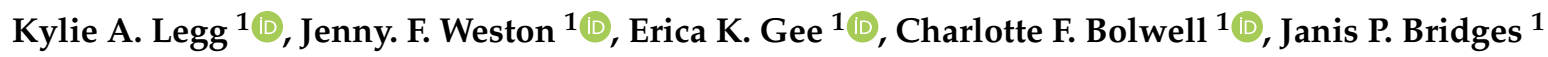 \\ and Chris W. Rogers $1,2, *$ (i) \\ 1 School of Veterinary Science, Massey University, Private Bag 11-222, 4442 Palmerston North, New Zealand \\ 2 School of Agriculture and Environment, Massey University, Private Bag 11-222, \\ 4442 Palmerston North, New Zealand \\ * Correspondence: C.W.Rogers@massey.ac.nz
}

Received: 2 August 2019; Accepted: 23 August 2019; Published: 27 August 2019

Simple Summary: International media has recently raised awareness about horse welfare during endurance competitions. However, much of this attention is focused on international level competitions (FEI) and little is known about domestic level competitions and their risk factors for elimination. The characteristics of endurance rides and risk factors for elimination of horses due to lameness and metabolic reasons were described by assessing the records of all competitors during six competition seasons in New Zealand (2010/11-2015/16). Endurance ride entries were dominated by lower distances $(40-80 \mathrm{~km})$, with the number of eliminations increasing with ride distance. The competition season was structured with the longer, more competitive rides at the end of the season, allowing the shorter, earlier rides to be used as conditioning rides. Ride distance, location and progression of the endurance season were significantly associated with eliminations due to lameness or metabolic reasons and horse age was significant for metabolic reasons only. The changing profile of endurance competitors over the years showed a decreasing number of higher level riders and subsequent increase in lower level riders competing in shorter rides. This profile with low competition speeds, demonstrates better horse welfare outcomes than seen in other parts of the world.

\begin{abstract}
The welfare of horses in endurance competitions has been the focus of recent media attention. Epidemiological studies have examined the sport at the international (FEI) level. However, much of the participation in the sport occurs at a national level in preparation for FEI level competition. The aims of this study were to describe participation in, and risk factors for elimination, from New Zealand endurance competitions. Data were collated from all endurance competitions $(\geq 40 \mathrm{~km})$ held in New Zealand during the 2010/11-2015/16 competition seasons. There were 6885 starts ( $\mathrm{n}=775$ horses, $\mathrm{n}=665$ riders), horses had a median age of 9 years (IQR 6.2-10.0) and had a median of 3 (IQR 2-5) starts per season. Accumulated ride distance per season per horse decreased from a median of $240 \mathrm{~km} /$ horse (IQR 120-440) in 2010/11 to $180 \mathrm{~km} /$ horse (IQR 80-320) in 2015/16. Ride entries were dominated by the $40 \mathrm{~km}(\mathrm{n}=2834,41 \%)$ and $80 \mathrm{~km}(\mathrm{n}=2517,37 \%)$ distances. Eliminations increased with ride distance, from $7 \%$ in $40 \mathrm{~km}$ rides to $53 \%$ in the $160 \mathrm{~km}$ rides. Lameness accounted for the majority of eliminations (64\%). The odds of elimination due to lameness were significantly associated with ride distance, location (North or South island) and time of year. The $11 \%$ of starters eliminated for metabolic reasons of the horse had increased odds of elimination associated with horse age, ride distance, location and time of year.
\end{abstract}

Keywords: horse; endurance; competition; elimination; lameness 


\section{Introduction}

Endurance rides are long distance competitions ridden over natural terrain, with distances ranging from $40 \mathrm{~km}$ to $160 \mathrm{~km}$. The horses are subjected to regular veterinary inspections at least every $40 \mathrm{~km}$ to ensure their fitness to start and to continue. Distances longer than $40 \mathrm{~km}$ are completed as multi-loop rides, with the expectation that an $80 \mathrm{~km}$ competition will be split into three loops and a $160 \mathrm{~km}$ competition will be run over 5-6 loops. Therefore there is an increasing frequency of veterinary inspection over the longer distances. In New Zealand, a qualification system is imposed on all horses and riders who are new to these competitions, even when the young or new horse is being ridden by an experienced rider [1]. This system limits the speed at which the horses can compete in the initial stages of their career (introductory or novice classes) to protect their welfare. The horse can then progress to intermediate and open level competitions. If a horse has been out of competition for more than two years, they drop to a lower level of competition and must prove themselves at that level before progressing.

The growing popularity of endurance riding worldwide has resulted in increased attention in the media regarding the welfare of endurance horses [2,3]. In particular, two different types of endurance riding seem to be evolving around the world; a more traditional endurance ride over challenging natural terrain where horses are commonly competing between 10 and $18 \mathrm{kmh}^{-1}$ and less technical courses where most horses race at $>20 \mathrm{kmh}^{-1}$ [4-6]. Epidemiological studies have examined the sport at Fédération Equestre Internationale (FEI) level events to identify risk factors contributing to elimination [7-10]. However, much of the participation in the sport occurs at a national level in preparation for FEI level sport [11,12], which may not reflect the same competition structure and risk factors characteristic of international level competition.

Internationally, elimination rates in endurance competitions range from $20 \%$ to $60 \%$ [8-10,13,14]. Lameness accounts for the majority of eliminations (50-70\%) with metabolic problems accounting for $15-25 \%$ of all eliminations $[10,13,14]$. Risk factors for elimination due to lameness or metabolic reasons are multifactorial and include: country, venue, number of entries in the competition, speed, ride length, track conditions, breed, age and horses' prior experience [6,7,13-15]. Other reasons for elimination include surface factors (injuries of the horse from the saddle, bridle or superficial leg injuries), course error, rider injury, rule violation and withdrawal from competition.

National (governed by Equestrian Sports New Zealand) and international level (FEI) endurance in New Zealand have been closely aligned for over 20 years [12]. The FEI have implemented new regulations to improve horse welfare based on data, with a focus on mandatory rest periods between successive competitions [16]. Further regulations are proposed to limit speed, at least in the early stages of the horses' international level competition. Recent studies of the characteristics of the endurance population in New Zealand have indicated that most participants are amateurs (i.e., they do not derive their main income from the sport) with a focus on participation, and who own and train their horses themselves [11,12]. The competition season in New Zealand begins in the spring (August/September) and ends in autumn (April/May), with the majority of riders in New Zealand using competitions as training opportunities for their horses [12]. It is possible that the risk factors for elimination may differ at national level competition, particularly when horses are competing at lower speeds. These competition characteristics suggest that international regulations pertaining to crewing, trainers and speed restriction [16] may not be necessary to protect the welfare of horses in the slower, more traditional endurance competitions that occur in New Zealand and some other countries.

The aim of this paper was to describe the participation in endurance competitions (domestic and FEI level) during six complete seasons in New Zealand. A secondary aim was to assess risk factors for elimination of the horses during these rides due to lameness or metabolic reasons.

\section{Materials and Methods}

All horses competing in official endurance rides within New Zealand have their entry and ride details logged with the official national registration body, Equestrian Sports New Zealand (ESNZ). 
These data are maintained as either paper copies (and loaded online as a PDF) or as a dynamic html table. Data were collated within a customised Access database from electronic and paper records of all Endurance competitions (minimum distance $40 \mathrm{~km}$ ) held within New Zealand during the 2010/11-2015/16 competition seasons. Ride details were cross referenced within the online ESNZ database to extract the demographic details for each horse (age, sex and previous cumulative kilometres in official rides). Horses that competed on a casual basis (single day registration) were excluded from further analysis in this study.

Ride distance was recorded according to the closest official ride category $(40-49 \mathrm{~km}=40 \mathrm{~km}$, $50-65 \mathrm{~km}=60 \mathrm{~km}, 78-88 \mathrm{~km}=80 \mathrm{~km}, 90-105 \mathrm{~km}=100 \mathrm{~km}, 120-124 \mathrm{~km}=120$ and $160 \mathrm{~km})$. Most ride distances were a multiple of $10 \mathrm{~km}$, although some events differed due to the difficulty in securing loops of specific distances.

Descriptive analysis was carried out for the variables of number of starts, completions, eliminations and ride speeds, and included calculation of the mean, median, IQR and minimal and maximal values. Normality was assessed using an Anderson-Darling test (for >5000 observations) with Pearson's Chi-squared test for differences between groups.

The exposure variables associated with ride elimination due to lameness reasons and due to metabolic reasons included horse-level variables; horse age and sex, as well as race-level variables; ride distance $(<100 \mathrm{~km}$ or $\geq 100 \mathrm{~km})$, competition season, competition level (national or international), rider age (junior or senior), rider gender, location (North or South Island of New Zealand) and month of year. Screening of all exposure variables for elimination due to lameness and elimination due to metabolic reasons was performed separately using univariable logistic regression. Variables with $p<0.2$ were considered for inclusion in the multivariate models. Exposure variables were then assessed for collinearity and those with the strongest association with the outcome variable were used in the final multivariate models. The multivariate models were built using a forward selection procedure whereby variables with a Wald-test $p \leq 0.05$ were retained in the model.

All statistical analyses were conducted using RStudio (version 3.5.1, 2018; R Foundation for Statistical Computing, Vienna, Austria), with the level of significance set at $p<0.05$.

\section{Results}

\subsection{Participants}

During the 2010/11-2015/16 competition seasons there were a total of 7491 starts in endurance rides of $\geq 40 \mathrm{~km}$ in New Zealand. Of the $92 \%(\mathrm{n}=6885)$ starts that had full registration details with ESNZ, the governing body for endurance in New Zealand, there were 775 horses and 665 riders. The remainder, $8 \%(n=606)$ of starts, were from 91 horses that competed on a casual day (single competition start) permit which restricted them to rides of $46 \mathrm{~km}$ or less [1] and were excluded from subsequent analysis. Of the horses registered with ESNZ, there were 425 (55\%) geldings, 338 (44\%) mares, $11(1 \%)$ stallions and 1 unrecorded, with a median age of 9 (IQR $=6-10)$ years.

\subsection{Starts}

Ride entries were dominated by the $40 \mathrm{~km}(\mathrm{n}=2834,41 \%)$ and $80 \mathrm{~km}(\mathrm{n}=2517,37 \%)$ distances, with $16 \%(n=1085)$ of all starts in competitions of $100 \mathrm{~km}$ or greater (Table 1$)$. There were fewer longer distance $(\geq 100 \mathrm{~km})$ races completed at the beginning of the endurance competition season than at the end of the season ( $5 \%$ vs. 33\%), reflecting the structure of the competition season. Median completion speed for all distances was higher for open horses than lower level horses with higher speeds observed for the longest distance rides $(160 \mathrm{~km})$ of $14.6 \mathrm{kmh}^{-1}$ (IQR 13.2-15.9 $\left.\mathrm{kmh}^{-1}\right)$. Across all seasons, the percentage of horses which were eliminated due to lameness or metabolic reasons increased with distance (Table 1). 
Table 1. Characteristics of competition distances over six endurance competition seasons 2010/11-2015/6 in New Zealand.

\begin{tabular}{|c|c|c|c|c|c|c|c|c|}
\hline & & \multicolumn{6}{|c|}{ Competition Distance (km) } & \multirow{2}{*}{$\frac{p}{\text { Value }}$} \\
\hline & & 40 & 60 & 80 & 100 & 120 & 160 & \\
\hline Horses & $\mathrm{n}$ & 729 & 236 & 529 & 226 & 199 & 103 & $<0.001$ \\
\hline Starts & $\mathbf{n}$ & 2834 & 449 & 2517 & 399 & 452 & 234 & $<0.001$ \\
\hline \multicolumn{9}{|l|}{ Completions: } \\
\hline $\begin{array}{l}\text { Beginning } \\
\text { (Aug-Nov) }\end{array}$ & $\begin{array}{c}n \\
(\%)\end{array}$ & $\begin{array}{l}1707 \\
(55 \%)\end{array}$ & $\begin{array}{c}154 \\
(5 \%)\end{array}$ & $\begin{array}{l}1100 \\
(35 \%)\end{array}$ & $\begin{array}{c}75 \\
(2 \%)\end{array}$ & $\begin{array}{c}95 \\
(3 \%)\end{array}$ & $\begin{array}{c}0 \\
(0 \%)\end{array}$ & $<0.001$ \\
\hline $\begin{array}{l}\text { Middle } \\
\text { (Dec-Feb) }\end{array}$ & $\begin{array}{c}\mathrm{n} \\
(\%)\end{array}$ & $\begin{array}{c}792 \\
(32 \%)\end{array}$ & $\begin{array}{l}200 \\
(8 \%)\end{array}$ & $\begin{array}{c}1016 \\
(40 \%)\end{array}$ & $\begin{array}{l}215 \\
(9 \%)\end{array}$ & $\begin{array}{l}210 \\
(8 \%)\end{array}$ & $\begin{array}{c}80 \\
(3 \%)\end{array}$ & $<0.001$ \\
\hline $\begin{array}{c}\text { End } \\
\text { (Mar-Jul) }\end{array}$ & $\begin{array}{c}\mathrm{n} \\
(\%)\end{array}$ & $\begin{array}{c}335 \\
(27 \%)\end{array}$ & $\begin{array}{c}95 \\
(8 \%)\end{array}$ & $\begin{array}{c}401 \\
(32 \%)\end{array}$ & $\begin{array}{l}109 \\
(9 \%)\end{array}$ & $\begin{array}{c}147 \\
(12 \%)\end{array}$ & $\begin{array}{c}154 \\
(12 \%)\end{array}$ & $<0.001$ \\
\hline Total & $\begin{array}{c}\mathrm{n} \\
(\%)\end{array}$ & $\begin{array}{l}2636 \\
(93 \%) \\
\end{array}$ & $\begin{array}{c}378 \\
(84 \%) \\
\end{array}$ & $\begin{array}{c}2101 \\
(84 \%) \\
\end{array}$ & $\begin{array}{c}283 \\
(71 \%) \\
\end{array}$ & $\begin{array}{c}292 \\
(65 \%) \\
\end{array}$ & $\begin{array}{c}109 \\
(47 \%) \\
\end{array}$ & $<0.001$ \\
\hline \multicolumn{9}{|l|}{$\begin{array}{c}\text { Speed } \\
\left(\mathrm{kmhr}^{-1}\right):\end{array}$} \\
\hline Novice/Int* & $\begin{array}{l}\text { Median } \\
\text { IQR }\end{array}$ & $\begin{array}{c}11.0 \\
9.7-12.3\end{array}$ & $\begin{array}{c}11.5 \\
10.3-12.8\end{array}$ & $\begin{array}{c}11.3 \\
10.3-12.3\end{array}$ & $\begin{array}{c}10.7 \\
10.0-11.3\end{array}$ & $\mathrm{n} / \mathrm{a}$ & $\mathrm{n} / \mathrm{a}$ & $<0.001$ \\
\hline Open * & $\begin{array}{c}\text { Median } \\
\text { IQR }\end{array}$ & $\begin{array}{c}12.7 \\
11.3-14.4 \\
\end{array}$ & $\begin{array}{c}13.7 \\
12.3-15.3 \\
\end{array}$ & $\begin{array}{c}13.2 \\
11.7-14.8 \\
\end{array}$ & $\begin{array}{c}13.6 \\
12.3-15.3 \\
\end{array}$ & $\begin{array}{c}13.7 \\
12.6-15.1 \\
\end{array}$ & $\begin{array}{c}14.6 \\
13.2-15.9 \\
\end{array}$ & $<0.001$ \\
\hline $\begin{array}{c}\text { Elimination } \\
\text { lameness }\end{array}$ & $\begin{array}{c}\mathrm{n} \\
(\%)\end{array}$ & $\begin{array}{c}92 \\
(3 \%)\end{array}$ & $\begin{array}{c}40 \\
(9 \%)\end{array}$ & $\begin{array}{c}267 \\
(11 \%)\end{array}$ & $\begin{array}{c}88 \\
(22 \%)\end{array}$ & $\begin{array}{c}100 \\
(22 \%)\end{array}$ & $\begin{array}{c}82 \\
(35 \%)\end{array}$ & $<0.001$ \\
\hline $\begin{array}{c}\text { Elimination } \\
\text { metabolic }\end{array}$ & $\begin{array}{c}\mathrm{n} \\
(\%)\end{array}$ & $\begin{array}{c}21 \\
(1 \%)\end{array}$ & $\begin{array}{c}10 \\
(2 \%)\end{array}$ & $\begin{array}{c}35 \\
(1 \%)\end{array}$ & $\begin{array}{c}6 \\
(2 \%)\end{array}$ & $\begin{array}{c}25 \\
(6 \%)\end{array}$ & $\begin{array}{c}22 \\
(9 \%)\end{array}$ & $<0.001$ \\
\hline
\end{tabular}

There were 46-68 competition events each season, with the 2012/13 season having the highest number of competitions and 2014/15 the lowest. There was a moderate decrease in the annual numbers of starts per season from 1271 in 2010/11 to 961 in 2015/2016. However, the number of open level competitors more than halved over the six seasons, with a concurrent increase in the number of intermediate and novice competitors (Table 2). This was reflected in the number of starters in the longer distance rides $(>100 \mathrm{~km})$ decreasing substantially over the six seasons (Table 2$)$. There was a decrease in the number of horses that had a start from 327 in 2010/11 to 286 in 2015/2016. The overall median number of starts per horse per season remained relatively constant at $3(\mathrm{IQR}=2-5)$, with a significant drop in starts per horse to a median of 2 (IQR $=1-4, p<0.001)$ in the 2014/5 season. $38 \%(n=297 / 775)$ of horses had starts in three or more seasons, with 44 horses $(6 \%)$ starting in all six seasons.

Table 2. Number of starters per season over six endurance competition seasons 2010/11-2015/6 in New Zealand.

\begin{tabular}{|c|c|c|c|c|c|c|}
\hline \multirow[b]{2}{*}{ Number of Starters } & \multicolumn{6}{|c|}{ Season } \\
\hline & $2010 / 2011$ & $2011 / 2012$ & $2012 / 2013$ & $2013 / 2014$ & $2014 / 2015$ & $2015 / 2016$ \\
\hline \multicolumn{7}{|l|}{ Distance $(\mathrm{km})$ : } \\
\hline 40 & 412 & 530 & 568 & 461 & 428 & 435 \\
\hline 60 & 71 & 67 & 82 & 113 & 41 & 75 \\
\hline 80 & 465 & 477 & 600 & 412 & 234 & 329 \\
\hline 100 & 138 & 75 & 89 & 39 & 30 & 28 \\
\hline 120 & 132 & 84 & 64 & 61 & 52 & 59 \\
\hline 160 & 53 & 29 & 50 & 29 & 38 & 35 \\
\hline Total & 1271 & 1262 & 1453 & 1115 & 823 & 961 \\
\hline \multicolumn{7}{|l|}{ Ride class: } \\
\hline Novice/Int * & 390 & 432 & 559 & 475 & 533 & 608 \\
\hline Open & 881 & 830 & 894 & 638 & 290 & 353 \\
\hline Horses (n) & 327 & 322 & 347 & 315 & 306 & 286 \\
\hline
\end{tabular}


The median accumulated ride distance per season per horse decreased from $240 \mathrm{~km} / \mathrm{horse}$ $(\mathrm{IQR}=120-440)$ in $2010 / 11$ to $180 \mathrm{~km} /$ horse $(\mathrm{IQR}=80-320)$ in $2015 / 16(p<0.001)$. Horses had a median of 22-28 days (IQR 8-71 and 14-50, respectively) between competitions following a $40 \mathrm{~km}$ race unless the next distance was $160 \mathrm{~km}$ where the median was 14 days (IQR 13-69). There was a median of 34-93 days following races $\geq 100 \mathrm{~km}$, generally with an increasing break following a larger first distance. There was a median of 63 days (IQR 55-90) between two consecutive $160 \mathrm{~km}$ rides.

\subsection{Risk Factors for Elimination}

During the 2010/11-2015/16 endurance seasons, $16 \%(n=1038)$ of horses failed to complete a ride. The percentage of horses that failed to complete a ride $\geq 100 \mathrm{~km}$ was $37 \%(\mathrm{n}=401 / 1085)$. There were 778 eliminations due to lameness or metabolic reasons, comprising $75 \%$ of all non-completions during the 2010/11-2015/16 endurance seasons. $64 \%(n=664)$ of all eliminations were due to lameness and $11 \%$ $(\mathrm{n}=110)$ were due to metabolic reasons. Univariable screening of variables identified five variables for inclusion $(p<0.2)$ in a multivariable model predicting elimination due to lameness (Table 3). These included horse age, ride distance $(<100 \mathrm{~km}$ or $\geq 100 \mathrm{~km}$ ), competition level (national or international FEI), location (North or South island of New Zealand) and month of year. Competition level had significant collinearity with ride distance $\left(\chi^{2}=3844, p<0.001\right)$ so was omitted from further analysis as ride distance had a stronger association with the outcome variable.

Table 3. Univariate logistic regression of associations between ride elimination ( $\mathrm{No} / \mathrm{Yes})$ due to lameness reasons only and the predictor variables; horse age and sex, ride distance $(<100 \mathrm{~km}$ or $\geq 100 \mathrm{~km})$, competition season, competition level (national or international), rider age (junior or senior), rider gender, location (North or South island of New Zealand) and month of year.

\begin{tabular}{|c|c|c|c|c|c|c|c|}
\hline \multirow[t]{2}{*}{ Variable } & \multirow[t]{2}{*}{ Category } & $\begin{array}{c}\text { Eliminated } \\
\text { No }\end{array}$ & $\begin{array}{c}\text { Eliminated } \\
\text { Yes }\end{array}$ & \multirow[t]{2}{*}{ OR } & \multirow[t]{2}{*}{$95 \% \mathrm{CI}$} & \multirow[t]{2}{*}{$p$} & \multirow[t]{2}{*}{$p$ (Wald) } \\
\hline & & n (\%) & n (\%) & & & & \\
\hline \multirow{3}{*}{ Horse age } & $\leq 8$ & 1871 (92\%) & $155(8 \%)$ & Ref & & & \multirow{3}{*}{$<0.001$} \\
\hline & $8-10$ & $1876(90 \%)$ & $216(10 \%)$ & 1.39 & $1.12-1.73$ & 0.003 & \\
\hline & $>10$ & $2465(89 \%)$ & $298(11 \%)$ & 1.46 & $1.19-1.79$ & $<0.001$ & \\
\hline \multirow{2}{*}{ Horse sex } & $\mathrm{F}$ & $2771(90 \%)$ & $304(10 \%)$ & Ref & & & \multirow{2}{*}{0.70} \\
\hline & M & 3444 (90\%) & $465(10 \%)$ & 0.97 & $0.82-1.13$ & 0.67 & \\
\hline \multirow{2}{*}{$\begin{array}{c}\text { Ride } \\
\text { distance }\end{array}$} & $<100 \mathrm{~km}$ & $5401(93 \%)$ & $399(7 \%)$ & Ref & & & \multirow{2}{*}{$<0.001$} \\
\hline & $\geq 100 \mathrm{~km}$ & $815(75 \%)$ & $270(25 \%)$ & 4.48 & $3.78-5.32$ & $<0.001$ & \\
\hline \multirow{6}{*}{$\begin{array}{l}\text { Competition } \\
\text { season }\end{array}$} & 2010/2011 & 1152 (91\%) & $119(9 \%)$ & Ref & & & \multirow{6}{*}{0.43} \\
\hline & 2011/2012 & 1149 (91\%) & $113(9 \%)$ & 0.91 & $0.71-1.17$ & 0.72 & \\
\hline & $2012 / 2013$ & 1305 (90\%) & $148(10 \%)$ & 1.04 & $0.82-1.32$ & 0.47 & \\
\hline & $2013 / 2014$ & 997 (89\%) & $118(11 \%)$ & 1.03 & $0.80-1.33$ & 0.32 & \\
\hline & $2014 / 2015$ & $754(92 \%)$ & $69(8 \%)$ & 1.04 & $0.78-1.37$ & 0.44 & \\
\hline & $2015 / 2016$ & $859(89 \%)$ & $102(11 \%)$ & 1.12 & $0.87-1.46$ & 0.33 & \\
\hline \multirow{2}{*}{$\begin{array}{c}\text { Competition } \\
\text { level }\end{array}$} & National & $5203(93 \%)$ & $372(7 \%)$ & Ref & & & \multirow{2}{*}{$<0.001$} \\
\hline & International & 1013 (77\%) & $297(23 \%)$ & 4.10 & $3.47-4.84$ & $<0.001$ & \\
\hline \multirow{2}{*}{ Rider age } & Junior & 790 (91\%) & $81(9 \%)$ & Ref & & & \multirow{2}{*}{0.87} \\
\hline & Senior & $5309(90 \%)$ & $560(10 \%)$ & 1.03 & $0.81-1.32$ & 0.82 & \\
\hline \multirow{2}{*}{ Rider gender } & F & 4758 (90\%) & $502(10 \%)$ & Ref & & & \multirow{2}{*}{0.41} \\
\hline & M & $1458(90 \%)$ & $167(10 \%)$ & 1.09 & $0.90-1.30$ & 0.38 & \\
\hline \multirow{2}{*}{$\begin{array}{l}\text { Location } \\
\text { (Island) }\end{array}$} & North & 4299 (91\%) & $422(9 \%)$ & Ref & & & \multirow[b]{2}{*}{0.001} \\
\hline & South & 1917 (89\%) & $247(11 \%)$ & 1.31 & $1.11-1.55$ & 0.001 & \\
\hline \multirow{12}{*}{ Month } & August & $276(98 \%)$ & $7(2 \%)$ & 0.41 & $0.17-0.83$ & 0.02 & \multirow{12}{*}{$<0.001$} \\
\hline & September & $688(96 \%)$ & $29(4 \%)$ & 0.67 & $0.43-1.04$ & 0.08 & \\
\hline & October & 1103 (94\%) & $69(6 \%)$ & Ref & & & \\
\hline & November & 879 (92\%) & $80(8 \%)$ & 1.45 & $1.04-2.04$ & 0.03 & \\
\hline & December & $880(89 \%)$ & $104(11 \%)$ & 1.89 & $1.38-2.60$ & $<0.001$ & \\
\hline & January & $740(86 \%)$ & $116(14 \%)$ & 2.51 & $1.84-3.44$ & $<0.001$ & \\
\hline & February & $601(89 \%)$ & $72(11 \%)$ & 1.92 & $1.36-2.71$ & $<0.001$ & \\
\hline & March & $591(86 \%)$ & $98(14 \%)$ & 2.65 & $1.92-3.68$ & $<0.001$ & \\
\hline & April & $291(78 \%)$ & $83(22 \%)$ & 4.56 & $3.23-6.45$ & $<0.001$ & \\
\hline & May & $94(91 \%)$ & $9(9 \%)$ & 1.53 & $0.69-3.01$ & 0.25 & \\
\hline & June & $42(95 \%)$ & $2(5 \%)$ & 0.76 & $0.12-2.54$ & 0.71 & \\
\hline & July & $31(100 \%)$ & $0(0 \%)$ & 0.00 & $0.00-1.02$ & 0.96 & \\
\hline
\end{tabular}


Univariable screening of variables identified six variables for inclusion $(p<0.2)$ in a multivariable model predicting elimination due to metabolic reasons (Table 4). These included horse age, ride distance $(<100 \mathrm{~km}$ or $\geq 100 \mathrm{~km}$ ), competition season, competition level (national or international FEI), location (North or South island of New Zealand) and month of year.

Table 4. Univariate logistic regression of associations between ride elimination (No/Yes) due to metabolic reasons only and the predictor variables; horse age and sex, ride distance $(<100 \mathrm{~km}$ or $\geq 100 \mathrm{~km}$ ), competition season, competition level (national or international), rider age (junior or senior), rider gender, location (North or South island of New Zealand) and month of year.

\begin{tabular}{|c|c|c|c|c|c|c|c|}
\hline \multirow[t]{2}{*}{ Variable } & \multirow[t]{2}{*}{ Category } & $\begin{array}{c}\text { Eliminated } \\
\text { No }\end{array}$ & $\begin{array}{c}\text { Eliminated } \\
\text { Yes }\end{array}$ & \multirow[t]{2}{*}{ OR } & \multirow[t]{2}{*}{$95 \% \mathrm{CI}$} & \multirow[t]{2}{*}{$p$} & \multirow[t]{2}{*}{$p$ (Wald) } \\
\hline & & n (\%) & n $(\%)$ & & & & \\
\hline \multirow{3}{*}{ Horse age } & $\leq 8$ & 2007 (99\%) & $19(1 \%)$ & Ref & & & \multirow{3}{*}{$<0.001$} \\
\hline & $8-10$ & 2058 (98\%) & $34(2 \%)$ & 1.75 & $1.00-3.13$ & 0.053 & \\
\hline & $>10$ & $2697(98 \%)$ & $66(2 \%)$ & 2.58 & $1.60-4.44$ & 0.000 & \\
\hline \multirow{2}{*}{ Horse sex } & $\mathrm{F}$ & 3024 (98\%) & $51(2 \%)$ & Ref & & & \multirow{2}{*}{0.758} \\
\hline & $\mathrm{M}$ & $3741(98 \%)$ & $68(2 \%)$ & 1.08 & $0.75-1.56$ & 0.688 & \\
\hline \multirow{2}{*}{$\begin{array}{c}\text { Ride } \\
\text { distance }\end{array}$} & $<100 \mathrm{~km}$ & $5734(99 \%)$ & $66(1 \%)$ & Ref & & & \multirow{2}{*}{$<0.001$} \\
\hline & $\geq 100 \mathrm{~km}$ & 1032 (95\%) & $53(5 \%)$ & 4.46 & $3.08-6.43$ & $<0.001$ & \\
\hline \multirow{6}{*}{$\begin{array}{l}\text { Competition } \\
\text { season }\end{array}$} & $2010 / 2011$ & $1245(98 \%)$ & $26(2 \%)$ & Ref & & & \multirow{6}{*}{0.021} \\
\hline & $2011 / 2012$ & 1244 (99\%) & $18(1 \%)$ & 0.69 & $0.37-1.26$ & 0.235 & \\
\hline & $2012 / 2013$ & 1434 (99\%) & $19(1 \%)$ & 0.63 & $0.34-1.15$ & 0.135 & \\
\hline & $2013 / 2014$ & 1103 (99\%) & $12(1 \%)$ & 0.52 & $0.25-1.02$ & 0.064 & \\
\hline & $2014 / 2015$ & 799 (97\%) & $24(3 \%)$ & 1.44 & $0.82-2.53$ & 0.205 & \\
\hline & $2015 / 2016$ & $941(98 \%)$ & $20(2 \%)$ & 1.02 & $0.56-1.83$ & 0.953 & \\
\hline \multirow{2}{*}{$\begin{array}{l}\text { Competition } \\
\text { level }\end{array}$} & National & $5514(99 \%)$ & $61(1 \%)$ & Ref & & & \multirow[b]{2}{*}{$<0.001$} \\
\hline & International & $1252(96 \%)$ & $58(4 \%)$ & 4.19 & $2.90-6.03$ & $<0.001$ & \\
\hline \multirow{2}{*}{ Rider age } & Junior & 859 (99\%) & $12(1 \%)$ & Ref & & & \multirow{2}{*}{0.530} \\
\hline & Senior & $5767(98 \%)$ & $102(2 \%)$ & 1.27 & $0.72-2.44$ & 0.443 & \\
\hline \multirow{2}{*}{ Rider gender } & $\mathrm{F}$ & $5171(98 \%)$ & $89(2 \%)$ & Ref & & & \multirow{2}{*}{0.758} \\
\hline & M & 1595 (98\%) & $30(2 \%)$ & 1.09 & $0.71-1.64$ & 0.677 & \\
\hline \multirow{2}{*}{$\begin{array}{l}\text { Location } \\
\text { (Island) }\end{array}$} & North & $4628(98 \%)$ & $93(2 \%)$ & Ref & & & \multirow{2}{*}{0.030} \\
\hline & South & $2138(99 \%)$ & $26(1 \%)$ & 0.61 & $0.38-0.92$ & 0.025 & \\
\hline \multirow{12}{*}{ Month } & August & 283 (100\%) & $0(0 \%)$ & 0.00 & $0-0.38$ & 0.972 & \multirow{12}{*}{$<0.001$} \\
\hline & September & 714 (100\%) & $3(0 \%)$ & 0.61 & $0.13-2.12$ & 0.468 & \\
\hline & October & $1164(99 \%)$ & $8(1 \%)$ & Ref & & & \\
\hline & November & $941(98 \%)$ & $18(2 \%)$ & 2.78 & $1.24-6.81$ & 0.017 & \\
\hline & December & 975 (99\%) & $9(1 \%)$ & 1.34 & $0.51-3.59$ & 0.545 & \\
\hline & January & $839(98 \%)$ & $17(2 \%)$ & 2.95 & $1.30-7.26$ & 0.012 & \\
\hline & February & $665(99 \%)$ & $8(1 \%)$ & 1.75 & $0.64-4.78$ & 0.265 & \\
\hline & March & $664(96 \%)$ & $25(4 \%)$ & 5.48 & 2.57-13.05 & 0.000 & \\
\hline & April & $348(93 \%)$ & $26(7 \%)$ & 10.87 & $5.10-25.89$ & 0.000 & \\
\hline & May & $98(95 \%)$ & $5(5 \%)$ & 7.42 & $2.21-22.68$ & 0.001 & \\
\hline & June & $44(100 \%)$ & $0(0 \%)$ & 0.00 & 0 -inf & 0.989 & \\
\hline & July & $31(100 \%)$ & $0(0 \%)$ & 0.00 & 0 -inf & 0.991 & \\
\hline
\end{tabular}

\subsection{Multivariable Logistic Regression}

The multivariable model identified increased odds of elimination due to lameness with longer distances, competition location in the South Island and progression of the competition season (Table 5). Competitions at the beginning of the competition season (August) had lower odds of elimination. 
Table 5. Multivariate logistic regression model of associations between ride elimination (No/Yes) due to lameness only and predictor variables; ride distance, location and time of year.

\begin{tabular}{cccccccc}
\hline Variable & Category & Estimate & SE & OR & 95\% CI & $p$ & $p$ (Wald) \\
\hline Intercept & & -2.84 & 0.13 & 0.06 & $0.04-0.07$ & $<0.001$ & \\
\hline Ride & $<100 \mathrm{~km}$ & Ref & & & & & $<0.001$ \\
distance & $\geq 100 \mathrm{~km}$ & 1.23 & 0.10 & 3.42 & $2.81-4.14$ & $<0.001$ & \\
\hline Location & North & Ref & & & & & \\
(island) & South & 0.24 & 0.09 & 1.27 & $1.07-1.52$ & 0.007 & \\
\hline & August & -0.87 & 0.40 & 0.42 & $0.17-0.86$ & 0.03 & \\
& September & -0.42 & 0.23 & 0.66 & $0.42-1.01$ & 0.06 & \\
& October & Ref & & & & & \\
& November & 0.03 & 0.18 & 1.03 & $0.73-1.46$ & 0.85 & \\
& December & 0.43 & 0.16 & 1.54 & $1.12-2.13$ & 0.008 & \\
& January & 0.43 & 0.17 & 1.54 & $1.11-2.14$ & 0.01 & $<0.001$ \\
& February & 0.22 & 0.18 & 1.25 & $0.87-1.79$ & 0.23 & \\
& March & 0.55 & 0.17 & 1.74 & $1.24-2.44$ & 0.001 & \\
& April & 0.72 & 0.19 & 2.05 & $1.24-3.00$ & $<0.001$ & \\
& May & 0.09 & 0.38 & 1.10 & $0.49-2.20$ & 0.80 & \\
& June & -0.20 & 0.73 & 0.82 & $0.13-2.74$ & 0.79 & \\
& July & -12.84 & 261.09 & 0.00 & $0.00-0.96$ & 0.96 & \\
\hline
\end{tabular}

The multivariable model identified increased odds of elimination due to metabolic reasons with horse age, longer distances, the North Island, November, and the end of the competition season (Table 6).

Table 6. Multivariate logistic regression model of associations between ride elimination (No/Yes) due to metabolic reasons only and predictor variables; horse age, ride distance, location and time of year.

\begin{tabular}{|c|c|c|c|c|c|c|c|}
\hline Variable & Category & Estimate & SE & OR & $95 \%$ CI & $p$ & $p$ (Wald) \\
\hline Intercept & & -5.24 & 0.41 & 0.01 & $0.00-0.01$ & $<0.001$ & \\
\hline \multirow{3}{*}{ Horse age } & $\leq 8$ & Ref & & & & & \multirow{3}{*}{$<0.001$} \\
\hline & $8-10$ & 0.29 & 0.29 & 1.34 & $0.76-2.43$ & 0.32 & \\
\hline & $>10$ & 0.67 & 0.27 & 1.96 & 1.18-3.39 & 0.01 & \\
\hline \multirow{2}{*}{$\begin{array}{c}\text { Ride } \\
\text { distance }\end{array}$} & $<100 \mathrm{~km}$ & Ref & & & & & \multirow{2}{*}{$<0.001$} \\
\hline & $\geq 100 \mathrm{~km}$ & 0.88 & 0.21 & 2.41 & $1.59-3.65$ & $<0.001$ & \\
\hline \multirow{2}{*}{$\begin{array}{l}\text { Location } \\
\text { (island) }\end{array}$} & North & Ref & & & & & \multirow{2}{*}{0.03} \\
\hline & South & -0.48 & 0.23 & 0.62 & $0.39-0.96$ & 0.04 & \\
\hline \multirow{12}{*}{ Month } & August & -13.57 & 385.16 & 0.00 & $0.00-0.35$ & 0.98 & \multirow{12}{*}{$<0.001$} \\
\hline & September & -0.44 & 0.68 & 0.65 & $0.14-2.25$ & 0.52 & \\
\hline & October & Ref & & & & & \\
\hline & November & 0.81 & 0.43 & 2.24 & $0.99-5.54$ & 0.06 & \\
\hline & December & 0.15 & 0.49 & 1.16 & $0.44-3.13$ & 0.76 & \\
\hline & January & 0.63 & 0.45 & 1.88 & $0.80-4.75$ & 0.16 & \\
\hline & February & 0.38 & 0.51 & 1.46 & $0.53-4.04$ & 0.46 & \\
\hline & March & 1.37 & 0.42 & 3.92 & 1.79-9.51 & 0.001 & \\
\hline & April & 1.74 & 0.44 & 5.70 & $2.51-14.23$ & $<0.001$ & \\
\hline & May & 1.59 & 0.59 & 4.89 & $1.42-15.29$ & 0.007 & \\
\hline & June & -13.70 & 977.56 & 0.00 & 0.00-inf & 0.99 & \\
\hline & July & -13.38 & 1164.31 & 0.00 & 0.00-inf & 0.99 & \\
\hline
\end{tabular}

\section{Discussion}

This was the first study to describe the participation and competition structure of endurance rides in New Zealand in addition to risk factors for elimination due to lameness or metabolic reasons. 
The majority of participants took part in 40 or $80 \mathrm{~km}$ rides with 2-3 starts per season. This profile represented the large proportion of amateur riders competing in the sport and the use of these lower distance rides as conditioning rides for FEI level events later in the season [12]. This is reflected by the greater number of longer $(\geq 100 \mathrm{~km})$ distance rides towards the end of the competition season (March-July) as compared to the beginning, reflecting the classic structure of the competition season in New Zealand with the pinnacle FEI rides and championship events towards the end of the season.

There was a decreasing number of open and thus longer distance starts over the six seasons, although the number of lower level competitors increased. This was supported by the decrease in median accumulated ride distance per season per horse across the six study seasons from $240 \mathrm{~km} /$ horse $-180 \mathrm{~km} /$ horse. The median number of starts per horse remained relatively constant across the seasons (with the exception of 2014/15), indicating that ride distances per horse decreased across the seasons. During the 2014/15 season, there were fewer competitions available, possibly accounting for the smaller number of starts per horse that season. The reasons for the decline in competitors in open and longer distance events are not clear. The introduction of mandatory rest periods in FEI endurance rules and these being incorporated into the ESNZ endurance rules $[1,16]$ may cause participants not to compete close to a championship event where elimination would cause them to become ineligible to compete at a pinnacle event. Sales of horses overseas and retirement of seasoned horses could be a contributing factor, as experienced riders developing a younger horse are ineligible to compete in the longer distances for several years. Increases in costs, both directly and indirectly associated with the sport (e.g., fuel and transport costs) may also impact riders' discretionary spending.

Completion speeds were at the lower end of international standards $\left(14-30 \mathrm{kmh}^{-1}\right.$ for international $160 \mathrm{~km}$ rides) $[7,8]$. These speeds could be affected by the use of rides as training opportunities [12] and the use of variable and hilly terrain for rides inherently providing a restriction on speed. Additionally, many events are held over farmland which may include a requirement to open and close gates whilst on course, further slowing speeds. Completion speeds increased with distance in the open category remaining constant across the middle distances. This indicated that the more experienced horses progressed from shorter conditioning rides early on in the season, to longer, faster and more competitive rides at the end of the season. The fact that championship events are held towards the end of the season further confounds these results as riders are more likely to try to be competitive when there is a national title at stake. The large number of novice riders in the lower distance rides maintained fairly constant speeds across the distances, remaining within their speed limitation of $13.5 \mathrm{kmh}^{-1}$ [1].

The temporal relationship between two consecutive starts indicated that riders entered into shorter distance competitions with a month or less until a second competitive ride. The length of time following longer distance rides was greater (1-3 months), indicating that more time off was allowed to horses having competed these rides (or that this was their pinnacle event). This aligned with the prescribed mandatory rest periods assigned to the different distances, with 5 and 12 days for 40 and $80 \mathrm{~km}$ rides respectively and increasing incrementally up to 33 days for rides $>146 \mathrm{~km} \mathrm{[1].} \mathrm{The}$ risk of elimination due to lameness has been shown to reduce with a periods of more than 90 days since a horses' previous FEI ride and the introduction of mandatory rest periods in the FEI rules have demonstrated clear benefits [15,17]. Data indicated that rest periods in New Zealand were more conservative than the guidelines ruled, but could be affected by rider location, competition schedule and adverse weather cancellations restricting competition opportunities.

\section{Elimination}

Elimination rates increased with increasing ride distance and speed in line with data published examining FEI rides [18]. The percentage of eliminated horses in the present study for rides $\geq 100 \mathrm{~km}$ $(37 \%)$ was less than that published in international studies of $\sim 50 \%[7,19]$. Unsurprisingly, the shorter length rides have a lower number of eliminations (total average elimination for all distances $=16 \%$ ), which was similar to elimination rates from national level rides in the USA $[8,13]$. This could be due to the aims of competitors in lower level competitions being for pleasure or training rather than to qualify 
or get the best possible placing. The percentage of horses eliminated for lameness or metabolic reasons was not significantly different across the six study seasons. This indicates that there has been little change in the competition or training practices across the study period. Therefore, this indicates that horse welfare has not been negatively compromised by inadequate guidelines as is suggested on an international scale [2]. In contrast to the increasing speed of top level competitions and subsequent increase in orthopaedic injuries reported internationally [4], competition speeds and elimination rates in New Zealand remained relatively constant across the six seasons.

A greater ride distance was likely to have a significant effect on eliminations for both lameness and metabolic reasons. This was in agreement with international studies $[7,13]$. Logically, a greater amount of exercise places an increased load on the musculoskeletal system (and respiratory system), and, coupled with muscular fatigue, would lead to increased risk of elimination due to lameness. In addition, longer rides were likely to be more competitive than the shorter distance rides used for preparation, possibly resulting in riders pushing their horses harder than they might during preparatory rides.

Location was associated with the risk of elimination due to both lameness and metabolic reasons. Horses competing in the South Island of New Zealand had a higher risk of elimination due to lameness than those in the North Island, which had a higher risk of elimination due to metabolic reasons. This may be attributable to a number of factors including terrain (South Island has rougher terrain), climate (warmer in the North Island) or training methods between the two islands, all of which are avenues for further investigation.

Time of year had a significant effect on the risk of elimination due to both lameness and metabolic reasons with the beginning of the season (August-October) having the lowest risk for both reasons. Risk of elimination due to lameness increased as the season progressed until April/May. This was likely an effect of the progressive loading of training and competitions throughout the season in addition to the higher number of horses starting in longer distance competitions later in the season. Furthermore, the summer months (November to March) coincide with warmer, dryer weather, resulting in hard ground, likely to increase the concussive forces on the horses' locomotor system. There was an increased risk of elimination due to metabolic reasons in November and March-May. This was likely due to the longer distance rides offered at these times of year, but could also reflect the advent of summer in November, and the beginning of cooler weather in March-May. The changing temperatures and increase of dust/pollen in the environment at these times of year may adversely affect the horses' respiratory systems. Additionally, the championship events (North Island, South Island and National Championships) include the majority of longer distance rides and are held between January and Easter. Riders are likely to ride more competitively and thus faster, at these events, and the higher elimination rates from these longer distance rides are more in line with those found in the international literature [10,14]. Longer distance rides also include a proportion of the event that is ridden in the dark, most commonly in the earlier stages of the ride, making it more difficult to judge the terrain and thereby increasing the risk of a horse becoming lame.

Risk of elimination due to metabolic reasons increased with increasing horse age, similar to previous studies $[9,13]$. This may be related to the minimum age limits set for competitions in New Zealand (minimum 6 years old for rides $\geq 100 \mathrm{~km}$ and 7 years for rides $\geq 140 \mathrm{~km}$ ) and a maximum speed restriction for 4 year old horses of $12 \mathrm{kmh}^{-1}$ [1]. For this reason, 8 years of age was chosen as the lower category range in the multivariate analysis. These restrictions may encourage more conservative racing strategies in younger horses and thus a lower risk of elimination for these horses.

\section{Conclusions}

Endurance competitions in New Zealand are attended by a diverse population of horses and riders, the majority of which participate in shorter distance rides, with slow speeds and few starts during the season. This reflects the amateur profile of New Zealand competitors and their use of shorter distance rides as conditioning rides for the more competitive, longer distance rides later in the season. The number of open level (and longer distance) competitors decreased over the study period, 
whilst the number of lower level competitors increased, reflecting the changing profile of the sport in New Zealand. Both speed and elimination rate increased with ride distance. Ride distance, location and month of year significantly affected risk of elimination due to lameness or metabolic reasons, whilst horse age was a significant factor for risk of elimination due to metabolic reasons only. This profile provides a basis for the adaptation of international regulations specific to endurance rides in New Zealand and confirms that endurance rides ridden at slower speeds over technically challenging terrain have fewer eliminations and better horse welfare.

Author Contributions: Conceptualization, C.W.R., C.F.B., E.K.G. and J.F.W.; methodology, C.F.B., C.W.R. and J.P.B.; formal analysis, K.A.L., J.P.B. and C.W.R.; data curation, K.A.L. and J.P.B.; writing-original draft preparation, K.A.L. and C.W.R.; writing-review and editing, all authors.

Funding: This study was funded by the New Zealand Equine Trust.

Acknowledgments: The authors would like to acknowledge the assistance of the following students in collation of the endurance ride records; Jessica Penders, Anne De Lannoy, Geordie Rogers, Julie Bates, Johanna Tait, Yuni Lin and Mia Payne.

Conflicts of Interest: The authors declare no conflict of interest. J.F.W. is involved in administration of endurance at a national level and is a FEI endurance veterinarian.

\section{References}

1. Equestrian Sports New Zealand. Competition Rules for the Equestrian Discipline of Endurance; Equestrian Sports New Zealand: Wellington, New Zealand, 2017.

2. Coombs, S.L.; Fisher, R.J. Endurance riding in 2012: Too far too fast? Veter. J. 2012, 194, 270-271. [CrossRef] [PubMed]

3. Marlin, D.J.; McEwen, J.; Sluyter, F. Completion and Treatment Rates in Modern Endurance Racing. In Proceedings of the 4th International Equitation Science Conference, Dublin, Ireland, 2-4 August 2008; p. 67.

4. Misheff, M.M.; Alexander, G.R.; Hirst, G.R. Management of fractures in endurance horses. Equine Veter. Educ. 2010, 22, 623-630. [CrossRef]

5. Adamu, L. Effects of Speed, Heart Rate, Lactate and Uric Acid on the Performance of Arabian Horses during a 120-Km Endurance Race. IOSR J. Agric. Veter. Sci. 2012, 1, 1-4. [CrossRef]

6. Bennet, E.; Parkin, T. Fédération Equestre Internationale endurance events: Risk factors for failure to qualify outcomes at the level of the horse, ride and rider (2010-2015). Veter. J. 2018, 236, 44-48. [CrossRef] [PubMed]

7. Nagy, A.; Murray, J.K.; Dyson, S.J. Descriptive epidemiology and risk factors for eliminations from Federation Equestre Internationale endurance rides due to lameness and metabolic reasons (2008-2011). Eq. Vet. J. 2014, 46, 38-44. [CrossRef] [PubMed]

8. Ii, H.C.S.; Marlin, D.J.; Geor, R.J.; Holbrook, T.C.; Deaton, C.M.; Vincent, T.; Dacre, K.; Schroter, R.C.; Jose-Cunilleras, E.; Cornelisse, C.J.; et al. Changes in selected physiological and laboratory measurements in elite horses competing in a $160 \mathrm{~km}$ endurance ride. Equine Veter. J. 2006, 38, 37-42. [CrossRef] [PubMed]

9. Adamu, L.; Adzahan, N.M.; Rasedee, A.; Ahmad, B. Physical Parameters and Risk Factors Associated with the Elimination of Arabian and Crossed Arabian Endurance Horses during a 120-km Endurance Race. J. Equine Veter. Sci. 2014, 34, 494-499. [CrossRef]

10. Dyson, S.; Nagy, A.; Murray, J.K. Elimination from elite endurance rides in nine countries: A preliminary study. Equine Veter. J. 2010, 42, 637-643.

11. Bolwell, C.F.; Rogers, C.W.; Rosanowski, S.M.; Weston, J.F.; Gee, E.K.; Gordon, S.J. Cross-Sectional Survey of the Management and Training Practices of Endurance Horses in New Zealand: A Pilot Study. J. Equine Veter. Sci. 2015, 35, 801-806. [CrossRef]

12. Webb, H.; Weston, J.; Norman, E.; Cogger, N.; Rogers, C. Experience, riding practices and training methods of Fédération Equestre Internationale (FEI: 80-160 km) level endurance horse rider-owner-trainers in New Zealand. Comp. Exerc. Physiol. 2019, 15, 137-145. [CrossRef]

13. Fielding, C.L.; Meier, C.A.; Balch, O.K.; Kass, P.H. Risk factors for the elimination of endurance horses from competition. J. Am. Veter. Med Assoc. 2011, 239, 493-498. [CrossRef] [PubMed]

14. Younes, M.; Barrey, E.; Cottin, F.; Robert, C. Elimination in long-distance endurance rides: Insights from the analysis of 7,032 starts in 80 to $160 \mathrm{~km}$ competitions. Comp. Exerc. Physiol. 2016, 12, 157-167. [CrossRef] 
15. Nagy, A.; Murray, J.K.; Dyson, S.J. Horse-, rider-, venue- and environment-related risk factors for elimination from Federation Equestre Internationale endurance rides due to lameness and metabolic reasons. Eq. Vet. J. 2014, 46, 294-299. [CrossRef] [PubMed]

16. FEI Endurance. Proposed Modifications to FEI Endurance Rules for 2020. FEI Endurance, Lausanne, Switzerland. 2019. Available online: https://inside.fei.org/system/files/MEMO\%20Endurance\%20v8_FOR\% 20PUBLICATION.pdf (accessed on 27 August 2019).

17. Bennet, E.D.; Parkin, T.D.H. The impact of the mandatory rest period in Federation Equestre Internationale endurance events. Eq. Vet. J. 2019. [CrossRef] [PubMed]

18. Bennet, E.D.; Parkin, T.D.H. Federation Equestre Internationale (FEI) endurance events: Riding speeds as a risk factor for failure to qualify outcomes (2012-2015). Vet. J. 2018, 236, 37-43. [CrossRef] [PubMed]

19. Burger, D.; Dollinger, S. Raisons d'élimination, état de santé et carrière sportive des chevaux dans les raids d'endurance en Europe et dans les pays arabes: Approche statistique. Prat. Vét. Eq. 1998, 30, 19-25.

(C) 2019 by the authors. Licensee MDPI, Basel, Switzerland. This article is an open access article distributed under the terms and conditions of the Creative Commons Attribution (CC BY) license (http://creativecommons.org/licenses/by/4.0/). 